\title{
A Soft Computing Decision Support System in the Diagnosis of Breast Cancer
}

\author{
Pankaj Srivastava \\ Department of Mathematics \\ MNNIT, Allahabad \\ 211004, U.P. India
}

\author{
Amit Srivastava \\ Applied Mechanics Department \\ MNNIT, Allahabad \\ 211004, U.P. India
}

\author{
Abdul Mannan \\ Applied Mechanics Department \\ MNNIT, Allahabad \\ 211004, U.P. India
}

\begin{abstract}
It is well known that most of Breast cancer diagnosis characterization processes are entirely based on physician's intuition and experience. Since diagnosis of breast cancer involves several layers of uncertainty and imprecision that makes traditional approaches inappropriate. In the present research paper a soft computing diagnostic support system for breast cancer is proposed which is capable enough to capture ambiguous and imprecise information prevalent in breast cancer diagnosis. It is user friendly and will sharpen diagnostic skill of medical practitioners.
\end{abstract}

\section{Keywords}

Soft Computing, Breast Cancer, Fuzzy Tools

\section{INTRODUCTION}

Breast cancer may be one of the oldest known forms of cancer in human. The oldest description of cancer was discovered in Egypt and dates back to approximately 1600 BC. Breast cancer is a disease of humans and other mammals while the overwhelming majority of cases in human are women [1]. Breast cancer is more than 100 times more common in women than breast cancer in men [2]. Prominent women who died of breast cancer include Empress Theodora, wife of Justinine; Anne of Austria, mother of Louis XIV of France; Mary Washington, mother of George and Rachel Carson, the environmentalist [1]. Janet Lane- Claypon conducted the first study on breast cancer epidemiology that has published in 1926 [1]. Worldwide breast cancer comprises $22.9 \%$ of all cancers in women. In 2008 breast cancer caused 458,253 deaths worldwide (13.7\% of cancer deaths in women) [3]. Landis et al. [4] reported that Breast cancer is most frequently diagnosed cancer and second leading cause of cancer deaths in women after Lung cancer. The latest report of NBCF, 2012 [5] reported that in 2010 approximately 207090 newly diagnosed case of Breast cancer and 30840 deaths due to Breast cancer in USA. Danaei et al. [6] found that $21 \%$ of all breast cancer deaths worldwide are attributable to alcohol use, overweight and obesity, and physical inactivity. This proportion was higher in high-income countries (27\%). In low- and middle-income countries, the proportion of breast cancers attributable to these risk factors was $18 \%$. IARC and Lacey et al. [7, 8] found that Breastfeeding has a protective effect. Clemons and Goss et al. [9] concluded that Estrogen through direct and indirect proliferative effects play role in development and growth of breast cancer. Prognosis and survival rates vary greatly depending on cancer type, staging and treatment and geographical location of the patient. Survival rates in the western world are very good [3]. Overall more than 8 out of 10 women in England that are diagnosed with the disease survive it for at least 5 years [10]. In the developing countries, however, survival rates are much poorer. Rao K. V. K et al. [11] discussed that despite advances in cancer treatment over the past decade, the prognosis of patients with breast cancer has improved only to small extent. Ford and Easton et al. [12] were of the view that hormonal imbalance and genetics are considered to be main risk of breast cancer.

Most of the real world problems that we are facing in day to day life are not ideal problems. These kinds of problems cannot be solved using the conventional way of computing but rather it is preferred to be solved using soft computing techniques [13]. Balanica, V. et al.[14] proposed a fuzzy logic technique for prediction of most probable risk estimation of breast cancer based on set of fuzzy rule that help in decision making process for the most suited treatment for a suspected breast cancer patient. Sipper et al. [15] examined the performance of fuzzy rule generation methods on Wisconsin breast cancer data (WBCD).WBCD problem combined with fuzzy genetic approach to evaluate system exhibit high classification performance. For accurate recognition of breast cancer tumour Khosravi et al. [16] proposed an automated and accurate hybrid system for identification of breast cancer. They have used fuzzy feature to increase the accuracy of their proposed system and this system tested against the data of WBCD. Oprea et al. [17] and Ekeh et al. [18] discussed about new segmentation technique for breast cancer detection based on the mammography. Cheng et al. [19] proposed a Fuzzy neural network approach to detect the malignant mass on mammograms which was somewhat useful for breast cancer control. Pandey, D. et al. [20] proposed a rule based system for cardiac analysis. Further Novruz Allahverdi et al. [21] proposed a fuzzy expert system to determination of coronary heart disease risk (CHD) of patient. Djam, X.Y. and Kimbi, Y.H. [22] developed a Fuzzy Expert System for the Management of Hypertension. Recently Srivastava, Pankaj and Srivastava, Amit [23, 24] proposed a soft computing diagnostic system to evaluate risk factor for coronary heart disease (CHD) and hypertension respectively. Srivastava, Pankaj et al [25] proposed a soft computing diagnostic system for the diagnosis of Hepatitis B. Srivastava, Pankaj and Sharma, Neeraja [26] proposed a soft computing decision support Diagnostic system for diabetes. Srivastava, Pankaj and Sharma, Neeraja [27] designed a soft computing diagnostic system that classify ECG beats in different phases and enables us to identify the status of cardiac health as per available ECG graphs. The present paper introduces a new soft computing model that measures risk factor on the basis of linguistic variables for the breast cancer. 


\section{METHODOLOGY}

In the present study input variables consist of Age, BMI, Age of first MC, Age of last MP, Age at first pregnancy, Alcohol intake, Smoking, Tumor surface area, Duration of breast feeding. Output variable consists of percentage of risk factor for Breast cancer.

\subsection{INPUT VARIABLES}

a) Age: Women current age is the best documented risk factor for breast cancer. The cumulative incidence of risk increase 1.8 percent at 50 year of age, 3.8 percent at 60 year age and 6.3 percent at 70 year age [28]. The incidence of breast cancer is very-very low before the age of 30 but after which it increase linearly up to the age of 80 [29]. The input variables for Age are classified in six fuzzy sets which as follows:

\begin{tabular}{|c|c|}
\hline \multicolumn{2}{|c|}{ Age (in years) } \\
\hline Young & $<30$ \\
\hline Aged young & $30-40$ \\
\hline Middle aged & $40-55$ \\
\hline Aged & $55-70$ \\
\hline Old & $70-85$ \\
\hline Very Old & $>85$ \\
\hline
\end{tabular}

$$
\begin{gathered}
\mu_{\text {young }}=\exp ^{((x-10) / 15)^{2} /(-2} \\
\mu_{\text {aged young }}=\exp ^{((x-40) / 10)^{2} /(-2} \\
\mu_{\text {middle aged }}=\exp ^{((x-55) / 15)^{2} /(-2} \\
\mu_{\text {aged }}=\exp ^{((x-70) / 15)^{2} /(-2} \\
\mu_{\text {old }}=\exp ^{((x-85) / 15)^{2} /(-2} \\
\mu_{\text {weryold }}=\exp ^{((x-95) / 10)^{2} /(-2}
\end{gathered}
$$

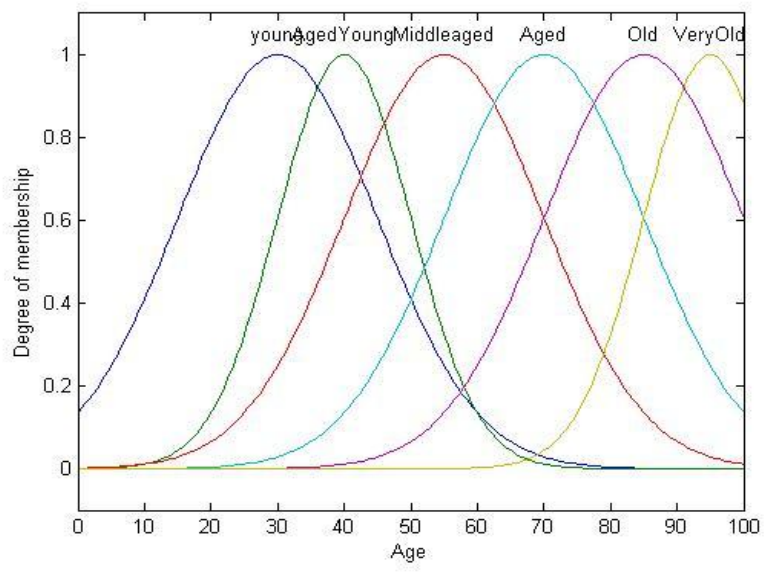

Figure1: Linguistic variables and membership function of Input variables 'Age'

b) Age of first menstrual cycle: Risk increase significantly with the age of menarche. If the first menstruated age is 15 or above 15 , risk factor decreases up to 23 percent than those having menarche prior to the age of 12[30]. The input variables for Age of first menstrual cycle are categorized in four fuzzy sets which are as follows:

\begin{tabular}{|c|c|}
\hline \multicolumn{2}{|c|}{ First menstrual cycle age (year) } \\
\hline Early & $10-12$ \\
\hline Normal & $12-14$ \\
\hline Above normal & $14-16$ \\
\hline Extreme & $\geq 16$ \\
$\mu_{\text {early }}=\exp ^{((x-12) / 2)^{2} /(-2}$ \\
$\mu_{\text {normal }}=\exp ^{((x-14) / 2)^{2} /(-2}$ \\
$\mu_{\text {above nomal }}=\exp ^{\left((x-16)^{2}\right)^{2} /(-2}$ \\
$\mu_{\text {extreme }}=\exp ^{((x-18) / 2)^{2} /-2}$
\end{tabular}

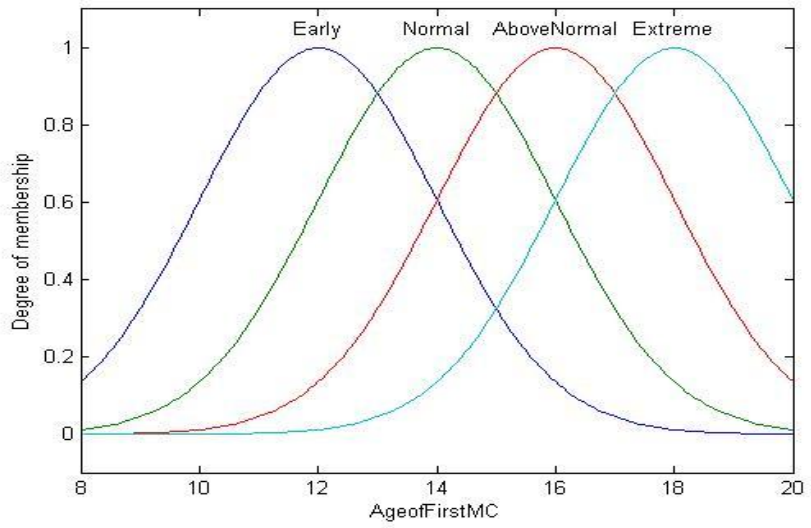

Figure2: Linguistic variables and membership function of Input variables 'First MC Age'

c) Age at last menstrual Period (LMP): At the age after 45 year the risk factor increase by 3 percent per year as the menopause is delayed. The increase in risk may be due to a longer lifetime exposure to the hormones estrogen and progesterone [31]. The input variables for Age of last menstrual period are classified in four fuzzy sets which are as follows:

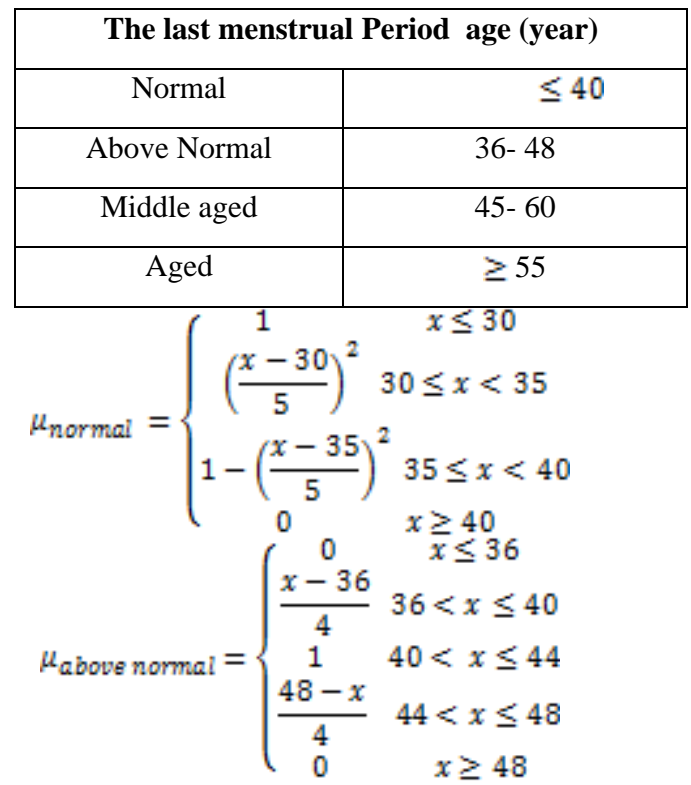




$$
\begin{gathered}
\mu_{\text {middle aged }}=\left\{\begin{array}{cc}
0 & x \leq 45 \\
\frac{x-45}{3} & 45<x \leq 48 \\
1 & 48<x \leq 56 \\
\frac{60-x}{4} & 56<x \leq 60 \\
0 & x \geq 60
\end{array}\right. \\
\mu_{\text {aged }}=\left\{\begin{array}{cc}
0 & x \leq 55 \\
\left(\frac{x-55}{3}\right)^{2} & 55 \leq x<58 \\
1-\left(\frac{x-58}{4}\right)^{2} & 58 \leq x<62 \\
1 & x \geq 62
\end{array}\right.
\end{gathered}
$$

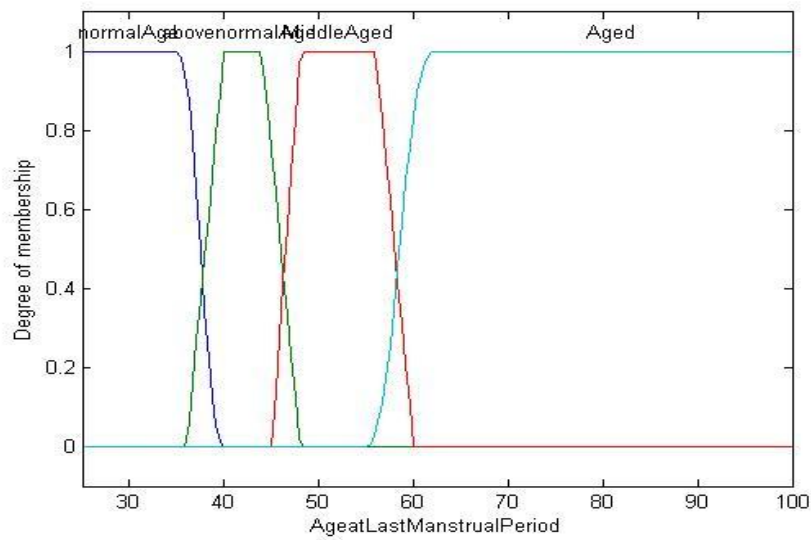

Figure3: Linguistic variables and membership function of Input variables 'last Menstrual Period age'

d) Age at First pregnancy: The women having pregnancy before 20 year of age have low risk of breast cancer in compare to the women having pregnancy after the age of 20 [32]. The input variables for Age at first pregnancy are classified in five fuzzy sets which are as follows:

\begin{tabular}{|c|c|}
\hline \multicolumn{2}{|c|}{ First pregnancy Age (years) } \\
\hline Early & $\leq 21$ \\
\hline Normal & $19-30$ \\
\hline Above normal & $28-40$ \\
\hline Middle aged & $38-48$ \\
\hline Aged & $\geq 45$ \\
$\mu_{\text {early }}=\left\{\begin{array}{cc}1 & x \leq 17 \\
\left(\frac{x-17}{2}\right)^{2} & 17 \leq x<19 \\
1-\left(\frac{x-19}{2}\right)^{2} & 19 \leq x<21 \\
0 & x \geq 21 \\
0 & x \leq 19 \\
\frac{x-19}{3} & 19<x \leq 22 \\
1 & 22<x \leq 27 \\
\frac{30-x}{3} & 27<x \leq 30 \\
0 & x \geq 30\end{array}\right.$
\end{tabular}
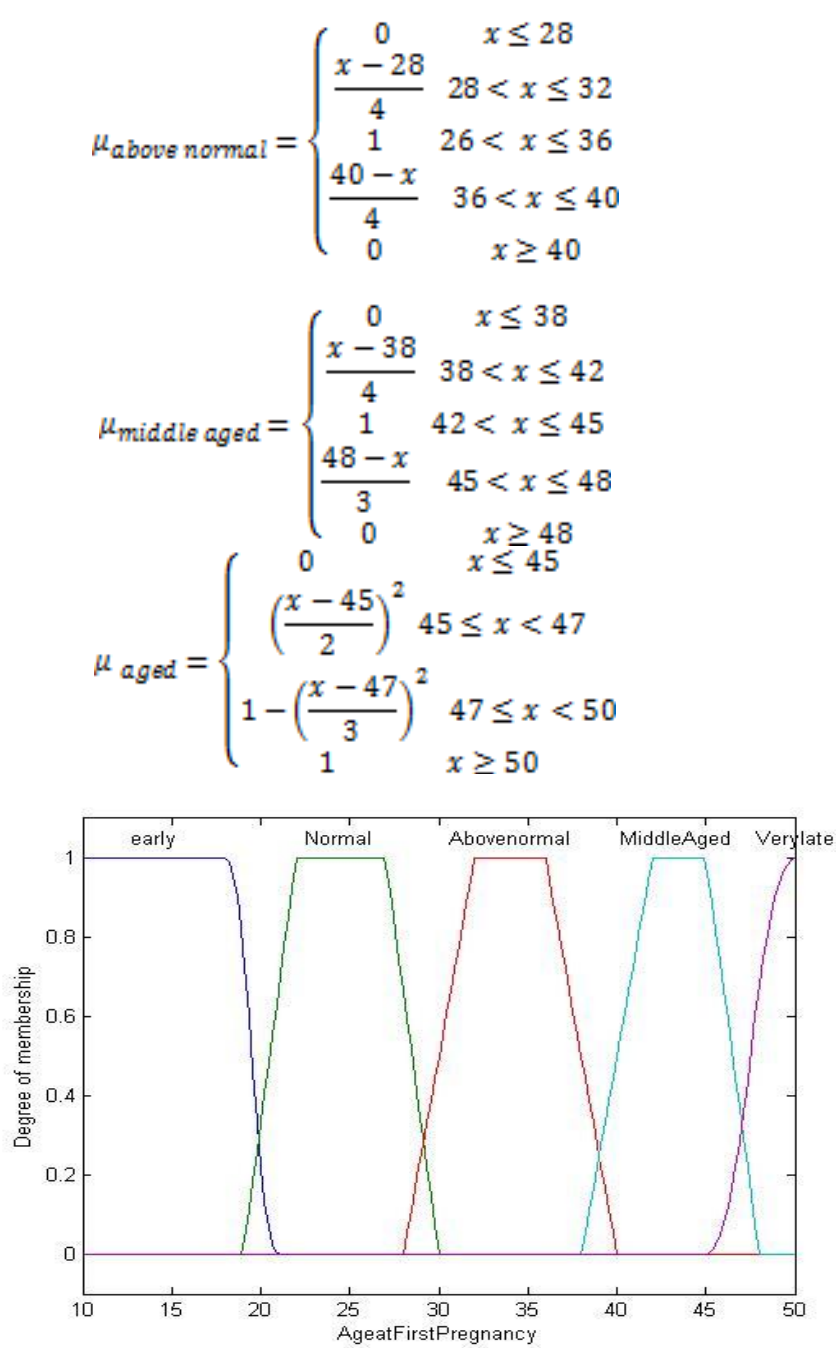

Figure4: Linguistic variables and membership function of Input variables 'First Pregnancy age'

e) Duration of breast feeding: Breast feeding protects women against developing breast cancer. Some studies have shown that breast-feeding slightly lowers breast cancer risk in women, especially in those women who had breast-feeding for $1 \frac{1}{2}$ to 2 years. As longer the women had breast feed to their child the risk of developing breast cancer decrease significantly [33]. The input variables for duration of breast feeding are categorized in six fuzzy sets which are as follows.

\begin{tabular}{|c|c|}
\hline \multicolumn{2}{|c|}{ Duration of breast feeding (months) } \\
\hline Very less & $\leq 4$ \\
\hline less & $2-8$ \\
\hline Normal & $6-14$ \\
\hline Above normal & $12-18$ \\
\hline High & $17-20$ \\
\hline Very high & $\geq 18$ \\
\hline
\end{tabular}




$$
\begin{aligned}
& \mu_{\text {veryless }}=\left\{\begin{array}{cc}
1 & x \leq 0 \\
\left(\frac{x-0}{2}\right)^{2} & 0 \leq x<2 \\
1-\left(\frac{x-2}{2}\right)^{2} & 2 \leq x<4 \\
0 & x \geq 4
\end{array}\right. \\
& \mu_{\text {less }}=\left\{\begin{array}{cc}
0 & x \leq 2 \\
\frac{x-2}{2} & 2<x \leq 4 \\
1 & 4<x \leq 6 \\
\frac{8-x}{2} & 6<x \leq 8 \\
0 & x \geq 8
\end{array}\right. \\
& \mu_{\text {normal }}=\left\{\begin{array}{cc}
0 & x \geq 8 \\
0 & x \leq 6 \\
\frac{x-6}{2} & 6<x \leq 8 \\
1 & 8<x \leq 12 \\
\frac{14-x}{2} & 12<x \leq 14 \\
0 & x \geq 14
\end{array}\right. \\
& \mu_{\text {above nomal }}=\left\{\begin{array}{cc}
0 & x \geq 14 \\
\frac{x-12}{2} & 12<x \leq 14 \\
1 & 14<x \leq 16 \\
\frac{18-x}{2} & 16<x \leq 18 \\
0 & x \geq 18
\end{array}\right. \\
& \mu_{\text {high }}=\left\{\begin{array}{cc}
0 & x \leq 17 \\
\frac{x-17}{2} & 17<x \leq 19 \\
1 & 19<x \leq 21 \\
\frac{2 a-x}{2} & 21<x \leq 23 \\
0 & x \geq 23 \\
0 & x \leq 21
\end{array}\right.
\end{aligned}
$$

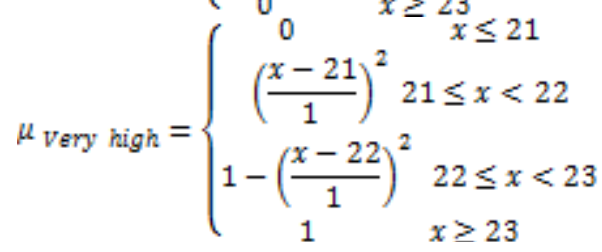

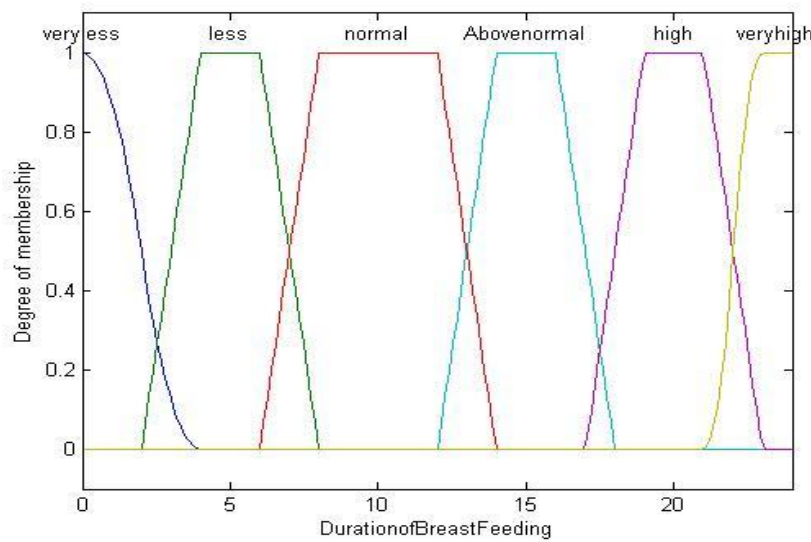

Figure5: Linguistic variables and membership function of Input variables 'Duration of Breast Feeding'

Alcohol Intake: Consumption of alcohol might increase the risk of breast cancer as it stimulates the metabolism of carcinogens such as acetaldehyde [34]. The input variables for alcoholic intake are classified in five fuzzy sets which are as follows:
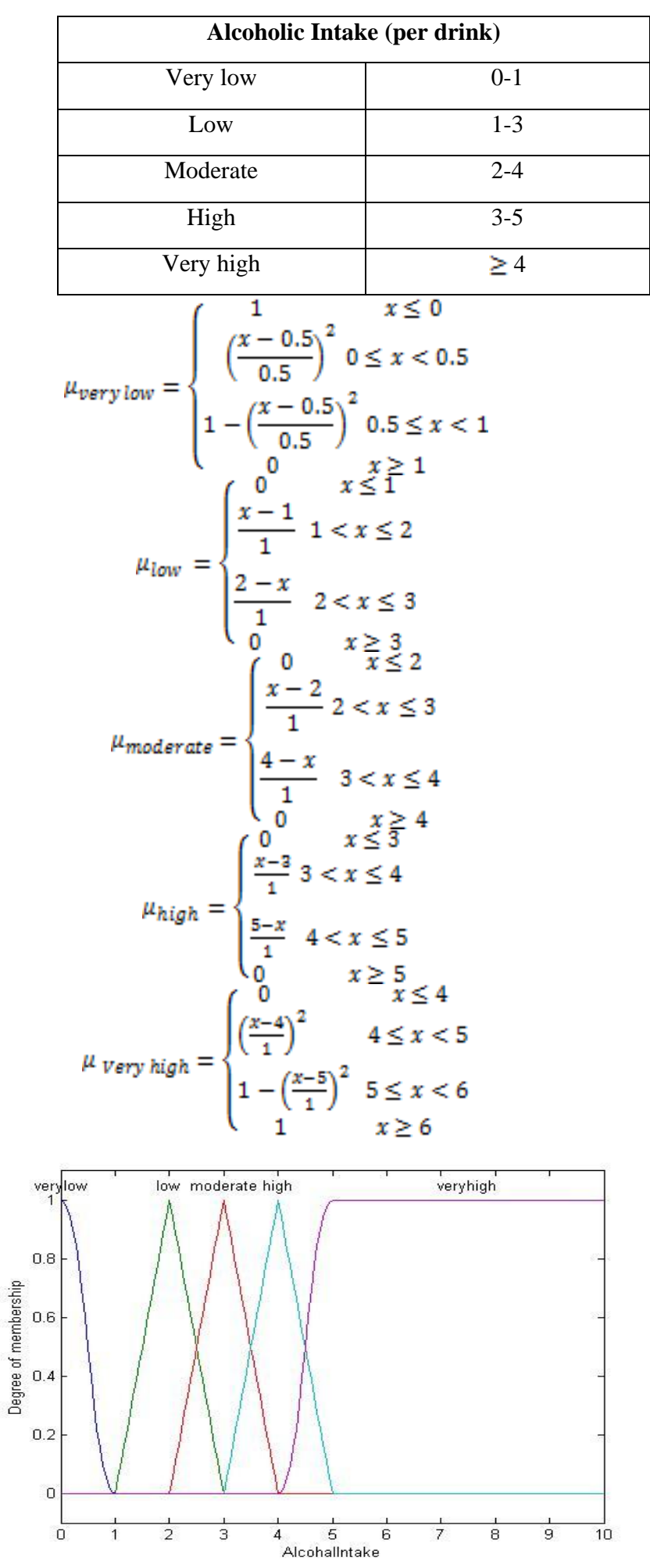

Figure6: Linguistic variables and membership function of Input variables 'Alcohol Intake'

g) Body Mass Index (BMI) of women: BMI of women after post menopause age increase the risk of breast cancer. Weight gain during the age of 18 to between the ages of 50 and 60 , has been consistently associated with risk of breast cancer after menopause [35]. The relative risk of breast cancer increase as we increase the intake of per drink (approximately 
$12 \mathrm{~g}$ alcohol) [36]. The input variables for body mass index are classified in to four fuzzy sets which are as follows:

\begin{tabular}{|c|c|}
\hline \multicolumn{2}{|c|}{ Body mass index $\left(\mathbf{k g} / \mathbf{m}^{2}\right)$} \\
\hline Low & $10-20$ \\
\hline Medium & $20-30$ \\
\hline High & $30-40$ \\
\hline Very high & $40-50$ \\
\hline
\end{tabular}

$\mu_{\text {low }}=\exp ^{((x-20) / 10)^{2} /(-2}$

$\mu_{\text {madium }}=\exp _{((x-30) / 10)^{2} /(-2}$

$\mu_{\text {high }}=\exp ^{((x-40) / 10)^{2} /-2}$

$\mu_{\text {veryhigh }}=\exp ^{((x-50) / 10)^{2} /-2}$

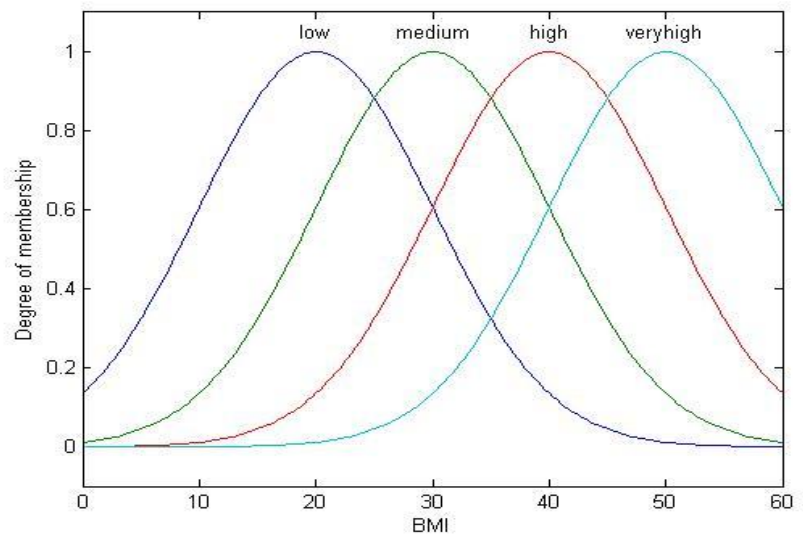

Figure7: Linguistic variables and membership function of Input variables 'BMI'

h) Tumor surface: The input variables for tumor surface are categorized in six fuzzy sets. which are as follows:

\begin{tabular}{|c|c|}
\hline \multicolumn{2}{|c|}{ Tumor surface Area (pixels) } \\
\hline Very small & $\leq 1000$ \\
\hline Small & $1000-2000$ \\
\hline Medium & $2000-3500$ \\
\hline Above medium & $3500-5000$ \\
\hline Large & $5000-6500$ \\
\hline Very large & $\geq 6500$ \\
\hline
\end{tabular}

$$
\begin{aligned}
& \mu_{\text {werysmall }}=\exp ((x-1000) / 500)^{2} /(-2 \\
& \mu_{\text {small }}=\exp ^{((x-2000) / 1000)^{2} / 2} \\
& \mu_{\text {medium }}=\exp ^{((x-1500) / 1500)^{2} /-2} \\
& \mu_{\text {above medium }}=\exp ^{((x-5000) / 1500)^{2} /(-2} \\
& \mu_{\text {large }}=\exp ^{((x-6500) / 1500)^{2} /-2} \\
& \mu_{\text {Lerylarge }}=\exp ^{((x-8000) / 1500) /(-2}
\end{aligned}
$$

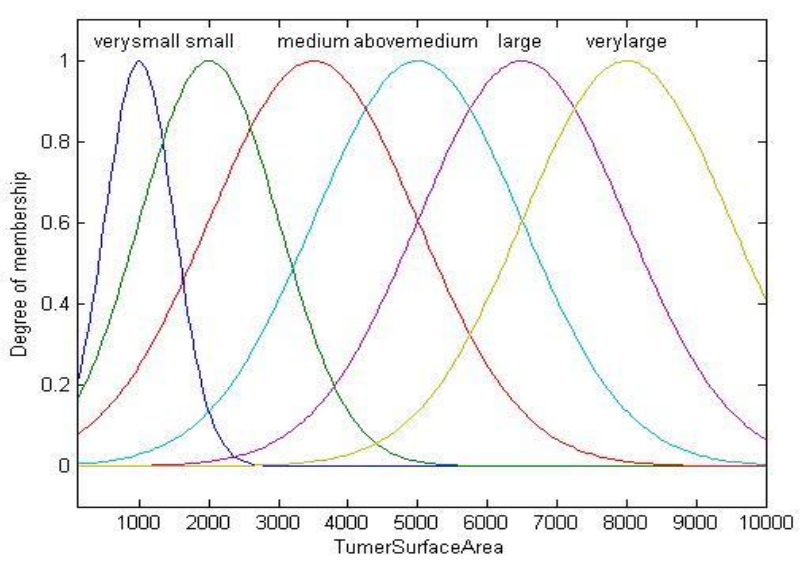

Figure8: Linguistic variables and membership function of Input variables 'Tumor Surface Area'

i) Smoking: The input variables for smoking are classified in five fuzzy sets which are as follows:

\begin{tabular}{|c|c|}
\hline \multicolumn{2}{|c|}{ Smoking } \\
\hline Very Low & $2-6$ \\
\hline Low & $4-12$ \\
\hline Moderate & $10-16$ \\
\hline High & $14-22$ \\
\hline Very high & $\geq 20$ \\
\hline
\end{tabular}

$$
\begin{gathered}
\mu_{\text {Verylow }}=\left\{\begin{array}{cc}
1 & x \leq 2 \\
\left(\frac{x-2}{2}\right)^{2} & 2 \leq x<4 \\
1-\left(\frac{x-4}{2}\right)^{2} & 4 \leq x<6 \\
0 & x \geq 6
\end{array}\right. \\
\mu_{\text {low }}=\left\{\begin{array}{cc}
0 & x \leq 4 \\
\frac{x-4}{2} & 4<x \leq 6 \\
1 & 6<x \leq 10 \\
\frac{12-x}{2} & 10<x \leq 12 \\
0 & x \geq 12
\end{array}\right. \\
\mu_{\text {high }}=\left\{\begin{array}{cc}
0 & x \leq 10 \\
\frac{x-10}{2} & 10<x \leq 12 \\
1 & 12<x \leq 14 \\
\frac{14-x}{2} & 14<x \leq 16 \\
0 & x \geq 16
\end{array}\right. \\
\mu_{\text {Very kigh }}=\left\{\begin{array}{cc}
0 & x \leq 14 \\
1-\left(\frac{x-22}{2}\right)^{2} & 22 \leq x<24 \\
\frac{x-14}{2} & 14<x \leq 16 \\
1 & 16<x \leq 20 \\
\frac{22-x}{2} & 20<x \leq 22 \\
0 & x \geq 22 \\
0 & x \geq 24 \\
\left(\frac{x-20}{2}\right)^{2} & 20 \leq x<22
\end{array}\right. \\
\end{gathered}
$$




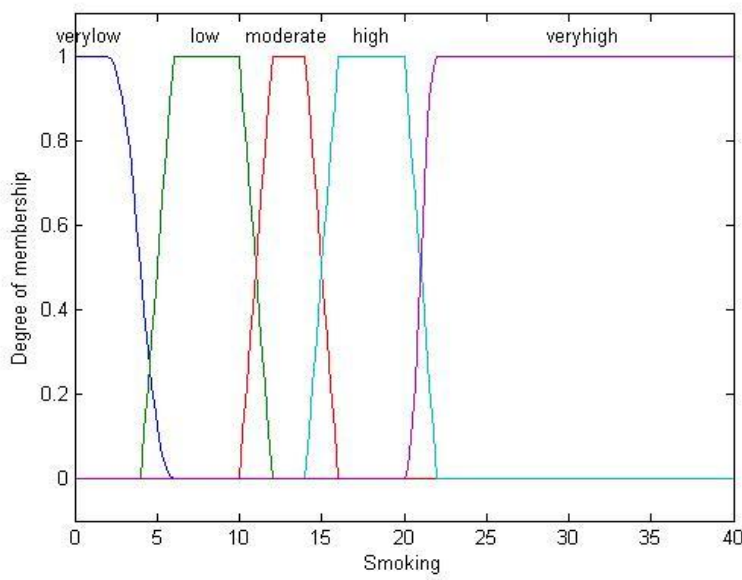

Figure9: Linguistic variables and membership function of Input variables 'Smoking'

\subsection{OUTPUT VARIABLE:}

The output zone reflects the percentage of risk factor for breast cancer in the patient under observation. It is classified in five layers such as; very less, less, medium, high and very high. Increasing order of percentage indicates increasing order of risk factor for breast cancer.

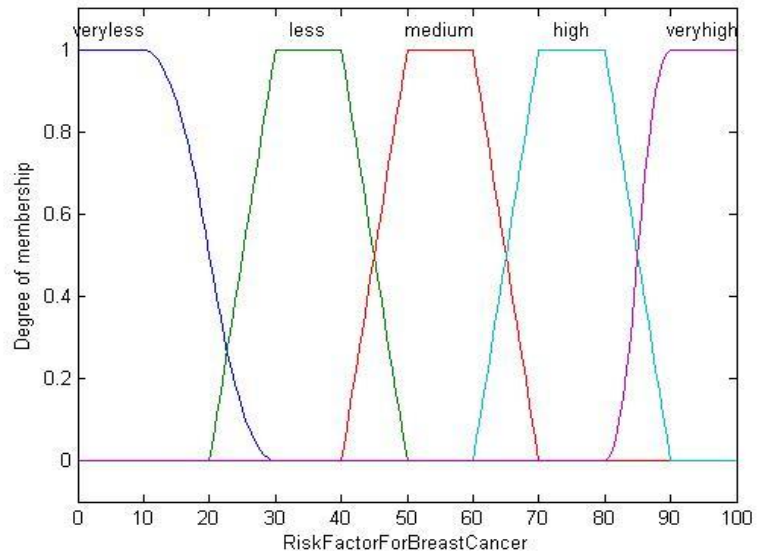

Figure10: Linguistic variables and membership function of Output variables 'Risk Factor for Breast Cancer'

\section{FUZZY RULES}

The proposed rule Base consists of a set of Fuzzy compositions and is derived from the Knowledge Base of Medical Experts. A fuzzy statement establishes a relationship between different input fuzzy sets and output sets. Some of the rules are given below in table. 


\begin{tabular}{|c|c|c|c|c|c|c|c|c|c|}
\hline \multicolumn{9}{|c|}{ Input Variables } & \multirow{2}{*}{$\begin{array}{l}\begin{array}{l}\text { Output } \\
\text { variable }\end{array} \\
\text { Risk } \\
\text { Factor }\end{array}$} \\
\hline $\begin{array}{l}\text { Age } \\
\text { (Y) }\end{array}$ & $\begin{array}{l}\text { BMI } \\
\left(\mathrm{kg} / \mathrm{m}^{2}\right)\end{array}$ & $\begin{array}{l}\text { First } \\
\text { menstrual } \\
\text { cycle } \\
\text { Age }(Y) \\
\end{array}$ & $\begin{array}{l}\text { Last } \\
\text { menstrual } \\
\text { Period } \\
\text { Age(Y) } \\
\end{array}$ & $\begin{array}{l}\text { First } \\
\text { pregnancy } \\
\text { Age (Y) }\end{array}$ & $\begin{array}{l}\text { Duration } \\
\text { of breast } \\
\text { feeding(mo } \\
\text { nth) }\end{array}$ & $\begin{array}{l}\text { Alcoholic } \\
\text { Intake(per } \\
\text { drink) }\end{array}$ & $\begin{array}{l}\text { Smoking } \\
\text { (cig.) }\end{array}$ & $\begin{array}{l}\text { Tumour } \\
\text { surface } \\
\text { (pixel) }\end{array}$ & \\
\hline Young & low & normal & normal & normal & Very high & no & no & Very small & Very less \\
\hline Young & low & normal & normal & normal & High & Very low & 2 & Very small & Very less \\
\hline ----- & ------ & $\begin{array}{l}----- \\
-4\end{array}$ & ------ & $\begin{array}{l}----- \\
-4\end{array}$ & ------ & ------- & $\begin{array}{l}---- \\
-4\end{array}$ & ------ & ------ \\
\hline young & medium & normal & normal & Ab. nor & High & Very low & 4 & Small & less \\
\hline ----- & ----- & ------ & ------ & ----- & ----- & - ---- & ---י- & ------ & ------ \\
\hline young & high & normal & Ab. nor & normal & High & low & 3 & medium & medium \\
\hline ----- & ---- & ------- & ------- & -------- & ------ & ------ & ---- & ------- & ------- \\
\hline $\begin{array}{l}\text { Aged } \\
\text { young }\end{array}$ & medium & Ab. normal & Normal & Ab. nor & Normal & low & 5 & medium & medium \\
\hline---- & ------ & ------ & ------ & ------ & ------ & ------- & ------ & ------ & ------ \\
\hline $\begin{array}{l}\text { Mid } \\
\text { aged }\end{array}$ & High & Ab. normal & Ab. normal & normal & Normal & low & 4 & $\begin{array}{l}\text { Above } \\
\text { medium }\end{array}$ & medium \\
\hline----- & ------ & ----- & ------ & ------ & ------ & -------- & ----- & ------ & ------ \\
\hline $\begin{array}{l}\text { Mid } \\
\text { aged }\end{array}$ & medium & Ab. normal & $\begin{array}{l}\text { Middle } \\
\text { aged }\end{array}$ & Ab. normal & Normal & moderate & 6 & $\begin{array}{l}\begin{array}{l}\text { Above } \\
\text { medium }\end{array} \\
\end{array}$ & medium \\
\hline----- & ------ & ----- & ------ & ----- & ------ & ------- & ----- & ------ & ----- \\
\hline $\begin{array}{l}\text { Mid } \\
\text { aged }\end{array}$ & High & Extreme & $\begin{array}{l}\text { Middle } \\
\text { aged }\end{array}$ & $\begin{array}{l}\text { Middle } \\
\text { aged }\end{array}$ & Low & high & 12 & large & High \\
\hline ------ & ------- & $\begin{array}{l}------ \\
\end{array}$ & ------ & ------- & --------- & ------- & ------- & ------- & ------- \\
\hline $\begin{array}{l}\text { Mid } \\
\text { aged }\end{array}$ & $\begin{array}{l}\text { Very } \\
\text { high }\end{array}$ & Extreme & Aged & Aged & Very low & v. high & 15 & Very large & Very high \\
\hline----- & ------ & ------ & ------ & ------ & ------ & ------- & ----- & ------ & ----- \\
\hline $\begin{array}{l}\text { Aged } \\
\text { young }\end{array}$ & low & normal & normal & normal & High & Very low & 4 & small & Very less \\
\hline ------- & ----- & ------- & -------- & -------- & ------ & -------- & ---- & ------ & ------- \\
\hline Aged & low & Normal & normal & Normal & High & Very low & 6 & small & less \\
\hline----- & ------ & ------ & ------ & ------ & ------ & ------- & ------ & ------ & ------ \\
\hline Aged & medium & Normal & Normal & Ab. normal & normal & high & 12 & medium & medium \\
\hline ----- & ------ & ------ & ------ & ------ & ------ & ------- & ----- & ------ & ------ \\
\hline Aged & medium & Ab. normal & Ab. normal & Normal & high & high & 1 & medium & medium \\
\hline----- & ------- & ------- & ------- & $\begin{array}{l}------ \\
\end{array}$ & ------ & ----- & ----- & ------- & ------- \\
\hline Aged & high & Ab. normal & Aged & Aged & Low & high & 10 & large & Very high \\
\hline ----- & ------ & ------ & ------ & ------ & ------ & ------- & ------ & ------ & ------ \\
\hline old & medium & normal & Ab. nor & normal & Very high & Very low & 3 & small & less \\
\hline ----- & ------ & ------ & ------ & ------ & ------ & ------- & ----- & ------ & ---- \\
\hline old & medium & Normal & Ab. normal & Ab. normal & Normal & high & 5 & medium & medium \\
\hline ----- & ------ & ------ & ------ & ------ & ------ & ------- & ------ & ------ & ----- \\
\hline old & medium & Ab. normal & Aged & Mid aged & Ab. normal & moderate & 8 & Medium & medium \\
\hline ----- & ------ & ------ & ------ & ------ & ------ & ------- & ------ & ------ & ------ \\
\hline $\begin{array}{l}\text { Very } \\
\text { old }\end{array}$ & high & Extreme & Mid aged & Mid aged & Low & high & 8 & large & High \\
\hline ------ & ------- & -------- & --------- & -------- & ------ & -------- & ------ & --------- & -------- \\
\hline $\begin{array}{l}\text { Very } \\
\text { old }\end{array}$ & $\begin{array}{l}\text { Very } \\
\text { high }\end{array}$ & Extreme & Aged & Aged & Very low & Very high & 12 & Very large & Very high \\
\hline
\end{tabular}

\section{DEFUZZIFICATION:}

Under the defuzzification process, rule base description of input variables following mamdani's approach was evaluated.

\section{RESULT:}

The 3D surface diagram designed on the basis of the rule base. It is clear from the following figure that high duration of breast feeding Vs. normal BMI indicates low risk factor of breast cancer whereas decreasing period of breast Feeding Vs. high BMI gives indication of high risk factor for breast cancer. Similarly number of observations can be made from various other $3 \mathrm{D}$ diagrams under different combinations of other input variables. 


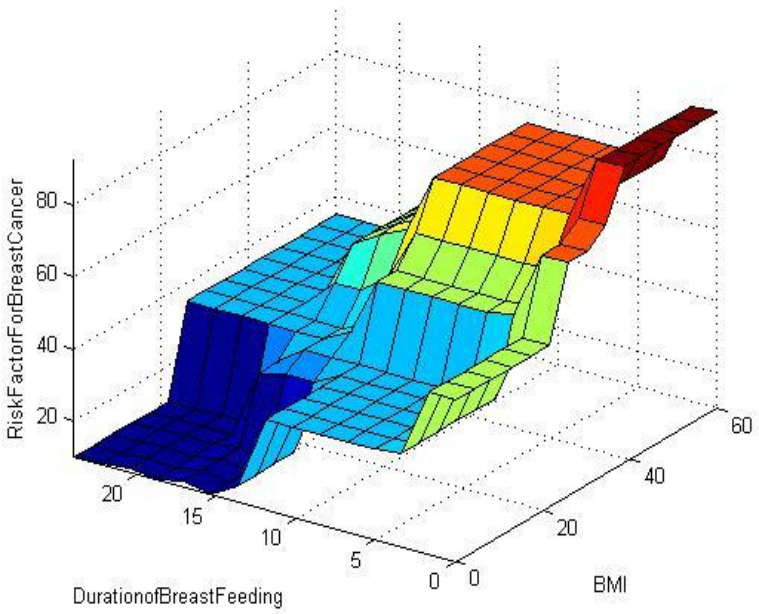

Fig 11- 3D surface view

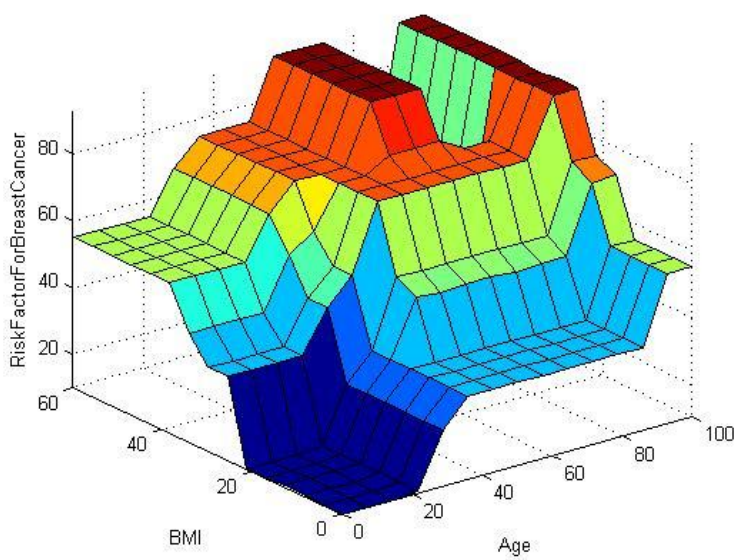

Fig 12- 3D surface view

\section{CONCLUSION:}

The present research article confirms that the soft computing models are competent enough to capture expert's knowledge and theoretical observations in a scientific way and handle real life problems in satisfactory manner. The proposed soft computing diagnostic system will be helpful for medical experts and it is user friendly that one can use itself.

\section{REFERENCES:}

[1] http://www.news-medical.net/health/History-of-BreastCancer.asp

[2] Male breast cancer treatment national cancer institute 2011

[3] World cancer report international agency for research on cancer 2008 .

[4] Landis, S. H., Murray, T., Bolden, S., and Wingo, P. A, (1999) "Cancer statistics" CA Cancer J. Clin., 49: 8-31

[5] National Brest Cancer Foundation, Inc, http//www.nationalbrestcancer.org

[6] Danaei G et al. (2005). Causes of cancer in the world: comparative risk assessment of nine behavioral and environmental risk factors. Lancet, 366, 1784-93.
[7] IARC (2008). "World cancer report 2008". Lyon, International Agency for Research on Cancer.

[8] Lacey JV Jr. et al. (2009). "Breast cancer epidemiology according to recognized breast cancer risk factors in the Prostate, Lung, Colorectal and Ovarian" (PLCO) Cancer Screening Trial Cohort. BMC Cancer, 9, 84.

[9] Clemons M, Goss P, (2011) "Estrogen and the risk of Breast cancer", The New England journal of medicine., 344: 276-285,

[10] ONS cancer survival in England, patient diagnosed 200408 followed up to 2009 , http://www.one.gov.uk/ons/publications/re-referencetables.html

[11] Indap.M.A., Radhika.S., Motiwale,L., Rao K.V.K., ( 2006) "Anticancer activity of phenolic antioxidants against Breast cancer cells and a spontaneous tumour.", Indian journal of pharmaceutical science,68(4), 470-474.

[12] Ford D and Easton D.F, (1995) "The genetics of breast and ovarian cancer", British Journal of Cancer 72, 805812

[13] Lotfi, A.Z., (1997) "The Roles of Fuzzy Logic and Soft Computing in the Conception, Design and Deployment of Intelligent Systems, in Software Agents and Soft Computing: Towards Enhancing Machine Intelligence, Concepts and Applications", Springer-Verlag.

[14] Victor Balanica, Ioan Dumitrache, Mihai Caramihai, William Rae, Charles Herbst, (2011) "Evolution of Brest Cancer Risk By Using Fuzzy Logic”, U.P.B. Sci. Bull., Series C, Vol. 73, Issue 1.

[15] Sipper M., Reyes C. A. P., (1999) "A fuzzy genetic approach to breast cancer diagnosis", Artificial Intelligence in Medicine 17 131-155.

[16] Khosravi A., Addeh J., Ganjipour J. (2011) "Breast cancer detection using BA-BP Based neural networks and efficient features. IEEE.

[17] Oprea A., Strungaru R., Ungureanu G. M., (2007)“New segmentation techniques for breast cancer detection based on mammography", 1st National Symposium on eHealth and Bioengineering, pp. 153-156.

[18] Ekeh A.P., Alleyne R. S., Duncan O. A., (2000) "Role of Mammography in diagnosis of breast cancer in inner-city hospital", Journal of the National Medical Association, 92:372-374.

[19] Cheng H.D., Cui M., (2004) "Mass lesion detection with a fuzzy neural Network", Pattern Recognition, 37, 6: 1189-1200.

[20] Pandey D., MahajanVaishali\&SrivastavaPankaj (2006) "Rule Based System for Cardiac Analysis", NATL ACAD SCI LETT, Vol. 29, No. 7\&8, pp 299-309

[21] Novruz ALLAHVERDI, Serhat and TORUN Ismail SARITAS, (2007) "Fuzzy Expert System Design for Determination of Coronary Heart Disease Risk" International Conference on Computer Systems and Technologies .

[22] Djam X.Y. and Kimbi Y.H., (2011) "Fuzzy Expert System for the Management of Hypertension" The Pacific Journal of Science and Technology Volume 12. Number 1.May 2011 (Spring)

[23] SrivastavaPankaj, SrivastavaAmit (2012) "A Soft Computing Approach for Cardiac Analysis" Journal of Basic and Applied Scientific Research, 2(1)376-385

[24] SrivastavaPankaj, SrivastavaAmit (2012) "Spectrum of Soft Computing Risk Assessment Scheme for Hypertension" International Journal of Computer Applications, Vol. 44- No17, pp 23-30

[25] SrivastavaPankaj, SrivastavaAmit, SirohiRitu (2012) "Soft Computing Tools and Classification Criterion for 
Hepatitis B" International journal of research and reviews in soft and intelligent computing, vol-2, No. 2

[26] SrivastavaPankaj and Sharma Neeraja (2012) "Soft computing decision support Diagnostic system for Diabetes" International Journal of Computer Applications, Volume 47- No.18

[27] SrivastavaPankaj and Sharma Neeraja (2012) " soft computing criterion for ECG beat classification and cardiac analysis" International Journal of Intelligent Systems (communicated)

[28] Steiner E., Klubert D., Knutson D.(2008) "Assessing Breast Cancer Risk in Women", American Family Physician,78(2008), 12

[29] Ries L. A. G., Eisner M. P., Kosary C. L., et al.(2000) (eds). SEER Cancer Statistics Review, 1973-1997, National Cancer Institute. NIH Pub. No. 00- 2789. Bethesda, MD,

[30] Brinton L. A., Schaiere C, Hoover R. N., (1988) "Menstrual factors and risk of breast cancer". Cancer Invest. 1988; 6: 145-154.

[31] McTiernan A, Kooperberg C, White E, et al, (2003) for the Women's Health Initiative Cohort Study.
Recreational physical activity and the risk of breast cancer in postmenopausal women. JAMA. 290(10):13311336.

[32] Lambe M, Hsieh C, Trichopoulos D, Ekbom A, Pavia M, Adami HO. (1994) "Transient increase in the risk of breast cancer after giving birth". N Engl J Med. 1994; 331(1):5-9.

[33] http://cancerhelp.cancerresearchuk.org/aboutcancer/cancer-questions/how-is-breast-feeding-relatedto-breast-cancer, 2012

[34] Vogel VG. (1998) "Breast cancer risk factors and preventive approaches to breast cancer". In: Kavanagh JJ, Singletary SE, Einhorn N, et al. (eds). Cancer in women. Malden, MA: Blackwell Science, 58-91.

[35] Andrew G Renehan, Margaret Tyson, Matthias Egger, Richard F Heller, and Marcel Zwahlen., (2008) "Bodymass index and incidence of cancer: a systematic review and meta-analysis of prospective observational studies", The Lancet; 371:569-578.

[36] Singletary S. E.,(2003) Rating the Risk Factors for Breast Cancer., Annals of surgery, 237: 4, 474-482 